\title{
Targeting the anti-inflammatory properties of the vagus nerve through vagus nerve stimulation: Therapeutic implications for inflammatory bowel diseases and other inflammatory conditions
}

\author{
B. Bonaz ${ }^{\bowtie 1,2}$ \\ ${ }^{1}$ Inserm, U1216, Grenoble Institute Neurosciences, University of Grenoble Alpes, \\ 38706 La Tronche Cedex, Grenoble, France \\ ${ }^{2}$ Division of Hepato-Gastroenterology, CHU Grenoble Alpes, 38700 La Tronche, Grenoble, France
}

\author{
Author \\ Bruno Bonaz, \\ Scopus AuthorID: 7004197628, \\ e-mail: BBonaz@chu-grenoble.fr

\section{For citation:} \\ Bonaz, B. \\ (2020) Targeting the anti- \\ inflammatory properties \\ of the vagus nerve through vagus \\ nerve stimulation: Therapeutic \\ implications for inflammatory bowel \\ diseases and other inflammatory \\ conditions. Integrative Physiology, \\ vol. 1 , no. 4 , pp. 277-284. \\ DOI: $10.33910 / 2687-1270-2020-1-$ \\ 4-277-284 \\ Received 29 September 2020; \\ reviewed 30 October 2020; \\ accepted 9 November 2020 \\ Copyright: () The Author (2020). \\ Published by Herzen State \\ Pedagogical University of Russia. \\ Open access under CC BY-NC \\ License 4.0.
}

\begin{abstract}
The vagus nerve (VN) is the longest nerve of the organism that innervates major organs such as the heart, lungs and gastro-intestinal tract and is a major component of the autonomic nervous system at the interface of the central nervous system and the body. The VN has anti-inflammatory properties both through its afferent fibers, activating the hypothalamic-pituitary adrenal axis, and its efferent fibers activating the cholinergic anti-inflammatory pathway. Targeting these anti-inflammatory pathways is of interest for various inflammatory conditions. Bioelectronic medicine through VN stimulation (VNS) appears as an interesting tool to release inflammatory conditions. VNS is approved for the treatment of drug-resistant epilepsy and has potential therapeutic applications in chronic inflammatory disorders such as inflammatory bowel diseases and others. Preclinical data and pilot clinical studies argue for such an effect. However, larger randomized double-blinded control study and a long-lasting follow-up of the patients to confirm these promising results are awaiting. In addition, the optimal neurostimulation parameters to better treat common conditions and diseases that involve immune regulation need to be determined.
\end{abstract}

Keywords: vagus nerve, vagus nerve stimulation, cholinergic anti-inflammatory pathway, hypothalamic-pituitary adrenal axis, inflammatory bowel diseases, rheumatoid arthritis.

\section{Introduction}

The vagus nerve $(\mathrm{VN})$, the tenth cranial nerve, is the longest nerve of the organism, which innervates major organs, such as the heart, lungs, and gastrointestinal tract. The vagus nerves are normally referred to in the singular. The $\mathrm{VN}$ is a major component of the parasympathetic nervous system, which, together with the sympathetic nervous system, forms the autonomic nervous system (ANS) at the interface of the central nervous system and the body. In this manuscript, we will focus on the $\mathrm{VN}$ distribution at the level of the digestive tract, as well as its anti-inflammatory properties, which can be targeted by vagus nerve stimulation (VNS) in the domain of inflammatory bowel diseases (IBD) and other inflammatory disorders.
The $\mathrm{VN}$ is a mixed nerve containing predominantly $80 \%$ of afferent fibers vehiculating visceral, somatic and taste sensations and $20 \%$ of efferent fibers controlling gastrointestinal motility and secretion. In particular, the VN is involved in the "conditioned reflex" described by the famous Russian researcher Ivan Petrovich Pavlov who won the Nobel Prize for Physiology or Medicine in 1904. The VN typically transmits information to the brain regarding luminal osmolarity, carbohydrate levels, mechanical distortion of the mucosa, and the presence of cytostatic drugs and bacterial products, whereas sympathetic afferents normally transmit visceral pain. Vagal efferents originate in the dorsal motor nucleus of the VN (DMNV), in the medulla, and innervate the digestive tract from the esophagus to the splenic flexure while the rest of the gut (left colon and rectum) is innervated 
by the sacral (S2-S4) parasympathetic nucleus (Netter, Colacino 1989). However, according to J. Delmas and G. Laux (1933), the VN innervates all the digestive tract in humans. In rats, S. M. Altschuler et al. (1993) reported that the VN innervates all of the digestive tract except the rectum. Vagal efferent fibers do not reach the intestinal lamina propria directly (Berthoud et al. 1991) but synapse onto enteric neurons that innervate the lamina propria where they release acetylcholine (ACh) acting on nicotinic or muscarinic receptors. Vagal afferent fibers originate from the mucosa to the muscle layers of the digestive tract. Their sensory afferent cell bodies are located in nodose ganglia and relay information to the nucleus tractus solitarii (NTS) (Cechetto 1987) and the area postrema, in close relation with the DMNV to form the dorsal vagal complex. Visceral information is then transmitted to areas of the forebrain such as the hypothalamus, amygdala, and cortex, via a relay through the parabrachial nucleus, hypothalamicpituitary-adrenal (HPA) axis, thalamus before final visceral afferent inputs in the insular cortex, the anterior cingulate and prefrontal cortices corresponding to the central autonomic network (CAN) (Benarroch 1993). The CAN, in turn, is able to modulate the ANS especially through projections of (i) the paraventricular nucleus of the hypothalamus $(\mathrm{PVH})$ to the DMNV and preganglionic neurons of the sympathetic nervous system at the spinal cord level; (ii) the amygdala to the DMN; (iii) the Barrington nucleus to the sacral parasympathetic nucleus; (iv) the A5 noradrenergic and $\mathrm{C} 1$ adrenergic groups to spinal preganglionic sympathetic neurons (Ricardo, Koh 1978). This complex is involved in the autonomic, endocrine and limbic responses of the "inner medium". The $\mathrm{VN}$ is a major component of interoceptive pathways and is considered the sixth sense of the body (Zagon 2001). Interoception, the sense of the body's internal physiological state, is regarded as the basis for motivational and emotional feelings and to the core neurobiological representation of self. Interoceptive mechanisms appear central to somatic disorders of brain-body interactions, including functional digestive disorders, such as irritable bowel syndrome (IBS), but also IBD (Bonaz et al. 2020; Fournier et al. 2020).

\section{Anti-inflammatory properties of the vagus nerve}

The VN has anti-inflammatory properties both through its afferent and efferent fibers (Fig. 1). Indeed, the release of peripheral pro-inflammatory cytokines, such as interleukin (IL)-1, Il-6, and tumour necrosis factor (TNF), by macrophages and other immune cells, following peripheral (iv or ip) injection of lipopolysaccharide (LPS), as a septic shock model (Werner et al. 2003), activates vagal afferents, through IL- $1 \beta$ receptors of the paraganglia. Then, the information is conveyed to the NTS, at the level of the medulla, where neurons projecting the information to the $\mathrm{PVH}$, around corticotrophinreleasing-factor (CRF)-containing neurons, are activated. These CRF neurons will then induce the release of adrenocorticotropic hormone by the pituitary, finally stimulating the release of glucocorticoids by the adrenal glands to dampen peripheral inflammation, i. e. the HPA axis. Therefore, the $\mathrm{VN}$ is a major component of the neuro-endocrine immune axis. This anti-inflammatory pathway is disrupted by vagotomy, which worsens experimental colitis (Ghia et al. 2006). Circulating pro-inflammatory cytokines are also able to target circumventricular organs, located outside the blood-brain barrier, that stimulate neighbouring neurons, which then stimulate the HPA axis (Buller 2001).

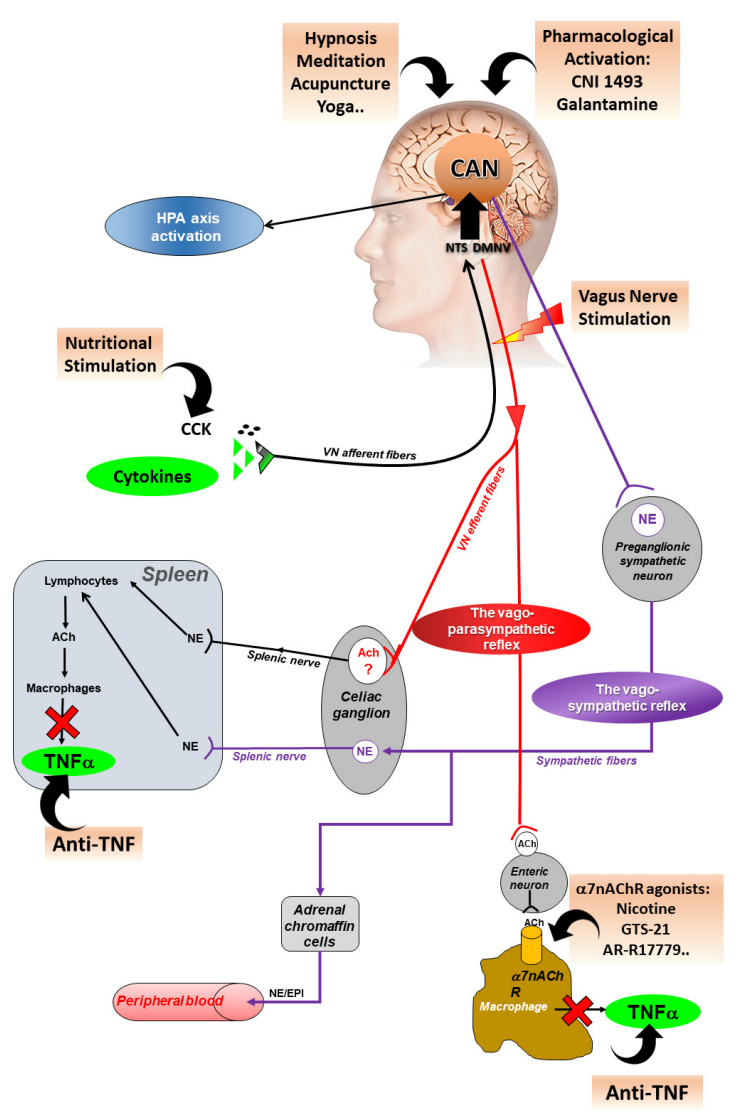

Fig. 1. Different pathways of the anti-inflammatory properties of the vagus nerve and their activation (from Bonaz et al. 2017). Ach, acetylcholine; CAN, central autonomic network; CCK, cholecystokinin; DMNV, dorsal motor nucleus of the vagus nerve; EPI, epinephrine; HPA, hypothalamic-pituitary-adrenal; NE, norepinephrine; NTS, nucleus tractus solitarius; $\mathrm{TNF} \alpha$, tumor necrosis factor-alpha; $\mathrm{VN}$, vagus nerve; $\alpha 7 \mathrm{nAChR}$, alpha7nicotinic acetylcholine receptor 
In 2000, Tracey's group described the parasympathetic cholinergic anti-inflammatory pathway (CAP) involving the vago-vagal reflex where vagal afferent fibers activate vagal efferent fibers (Fig. 1). Indeed, peripheral iv injection of LPS inducing a septic shock in rats was prevented by $\mathrm{VN}$ stimulation (VNS) of the distal end cut VN, thus stimulating vagal efferent fibers (Borovikova et al. 2000). This effect was mediated by the release of ACh by the $\mathrm{VN}$ inhibiting the release of pro-inflammatory cytokines such as TNF by activated macrophages. Besides TNF, other pro-inflammatory cytokines such as IL6 and IL1 $\beta$ were significantly decreased by VNS, but not the anti-inflammatory cytokine IL-10. This anti-inflammatory effect was mediated by linking ACh with $\alpha-7$-nicotinic ACh receptors ( $\alpha 7 \mathrm{nAChR})$ of macrophages and was suppressed in $\alpha 7 \mathrm{nAChR}$ knockout animals (Wang et al. 2003). The intra-cellular signaling of $\alpha 7 n A C h R s$ inhibits transactivational activity of the transcription factor NF-kB p65 (Wang et al. 2004) and activates Jak2 and STAT3 signalling (de Jonge et al. 2005). However, the $\mathrm{VN}$ does not interact directly with resident macrophages in the gut but interacts with nNOS, VIP, and ChAT enteric neurons located within the gut muscularis and with their nerve endings close to resident macrophages (Cailotto et al. 2014).

Tracey's group also described a vago-sympathetic anti-inflammatory pathway through which the $\mathrm{VN}$ activates the splenic sympathetic nerve (Rosas-Ballina et al. 2008) (Fig. 1). Indeed, the release of norepinephrine by this splenic nerve binds to the $\beta 2$ adrenergic receptor of splenic lymphocytes, which release $\mathrm{ACh}$, which binds to $\alpha 7 \mathrm{nAChRs}$ of splenic macrophages finally inhibiting the release of TNF by the spleen (Olofsson et al. 2012). This is the non-neuronal cholinergic pathway. Thus, the VN has an anti-TNF effect either at the peripheral macrophages level or at the spleen level. However, for some authors, the efferent pathway of the CAP is not the $\mathrm{VN}$ but the sympathetic nervous system. For example, D. Martelli et al. (2014) proposed a non-neural link from the VN to the spleen where $\alpha 7 \mathrm{nAChRs}$ are located on the peripheral terminals of the splenic sympathetic nerves. When stimulated by ACh from incoming T-cells, these terminals release norepinephrine, which then acts on $\beta$ adrenergic receptors on splenic macrophages to suppress their release of TNF. Another anatomical pathway could be the activation of the celiac ganglion, at the origin of the innervation of the spleen, by brain nuclei that are parts of the CAN and that generate patterns of autonomic responses via projections to preganglionic sympathetic neurons in the spinal cord, such as the $\mathrm{C} 1$ adrenergic group (Abe et al. 2017). Indeed, five cell groups in the brain regulate the entire sympathetic outflow (Strack et al. 1989): the PVN, the A5 noradrenergic cell group, the caudal raphe region, the rostral ventrolateral medulla ( $\mathrm{C} 1$ adrenergic group), and the ventromedial medulla. The activation of the afferent arm (i. e. vagal afferents) of the inflammatory reflex could activate the CAN, through projections from the NTS, to modulate the sympathetic nervous system through these five cell groups. In this case, the $\mathrm{VN}$ would induce an indirect anti-inflammatory reflex by activating the sympathetic nervous system.

Thus, the anti-inflammatory pathways involving the $\mathrm{VN}$ are rather complex and it clearly appears that both vagal afferents and efferents are of interest in this respect. Targeting this anti-inflammatory pathway is of interest in various pathologic conditions, especially in inflammatory disorders where cytokines are involved in their pathogeny such as IBD, represented by Crohn's disease (CD) and ulcerative colitis, and rheumatoid arthritis (RA). Presently, in the domain of IBD and RA, biological therapies, such as anti-TNF, have revolutionized the care of these patients. However, these treatments have complex side effects (Click, Regueiro 2019) resulting in $30-50 \%$ of non-adherence (Chan et al. 2017); and patients show a growing interest in complementary medicines (Torres et al. 2019). These biological therapies act downstream of macrophages and other immune cells while other therapeutic approaches can act upstream (Fig. 1). For example, (i) $\alpha 7 \mathrm{nAChRs}$ agonists (GTS-21, ARR17779) or nicotine have been used in inflammatory models of post-operative ileus, pancreatitis; (ii) activation of the central cholinergic pathway with CNI 1493 or Galantamine in a model of carrageenan-induced paw edema and endotoxemia improve inflammation; (iii) nutritional therapy with fat nutrition induces the release of cholecystokinin which activates vagal afferents which blunted hemorrhagic shock-induced TNF and IL-6 release; (iv) complementary therapies such as hypnosis, meditation, tai chi, acupuncture as well as physical activity stimulate the $\mathrm{VN}$; and, finally, (v) VNS is of interest in activating the CAP; we will now focus on this non-drug therapy approach (see a review by Bonaz et al. 2017; 2019).

\section{Vagus nerve stimulation in epilepsy}

VNS was approved by the FDA for the treatment of drug-resistant epilepsy in 1997 (Bonaz et al. 2013). Today, $\sim 100,000$ patients have been implanted for epilepsy. About $50 \%$ of patients have a significant reduction in seizure frequency, with $\sim 12 \%$ experiencing a $90 \%$ decrease in seizures 
(Englot et al. 2011). VNS is a slow acting therapy because its effect needs some latency in epilepsy and its effectiveness improves over time, according to a 3-year follow-up study (Morris et al. 1999). Although the anti-epileptic effect of VNS was supposed to be related to vagal C-fibres, their destruction did not alter subsequent VNS-induced seizure suppression in rats; thus arguing for an involvement of vagal A- and B-fibres (Krahl et al. 2001). VNS is a safe procedure with the most adverse events reported being hoarseness (20-28\%), paraesthesia $(12 \%)$, headache $(4.5 \%)$, and shortness of breath (3.2\%) (Morris et al. 1999) usually occurring during stimulation and often decreasing over time and/or following the modification of stimulation parameters such as intensity and pulse width. No significant impact on heart rate has been reported. Indeed, VNS is performed on the left VN, innervating the atrioventricular node (regulating the force of contraction of the heart muscle with less influence on the heart rate) while the right $\mathrm{VN}$ innervates the sinoatrial node (involved in the pace-making function of the heart) (Bonaz et al. 2013). VNS parameters activating vagal afferents in epilepsy are: frequency, $20-30 \mathrm{~Hz}$; intensity, $0.5-1.5 \mathrm{~mA}$; pulse width, $500 \mu \mathrm{s}$; on-time, $30 \mathrm{~s}$; and off-time, $5 \mathrm{~min}$; they are easily adjusted with a programming wand (Bonaz 2013). VNS in epilepsy is normally invasive, requiring a $\sim 1 \mathrm{~h}$ surgery performed under general anaesthesia usually by a neurosurgeon familiar with this technique. The electrode (Model 302, Livanova, London) is wrapped around the left $\mathrm{VN}$ at the cervical level near the carotid artery, tunnelled under the skin and connected to a bipolar pulse generator (Model 102) implanted subcutaneously in the left chest wall.

In epilepsy treatment, VNS aims to stimulate vagal afferents at a high frequency of $20-30 \mathrm{~Hz}$, while for activating the CAP vagal efferents need to be stimulated at a low-frequency $(1-10 \mathrm{~Hz})$ (Borovikova 2000; Bernik et al. 2002). However, we have shown that even at a low frequency stimulation of $5-10 \mathrm{~Hz}$, VNS also activates vagal afferents targeting the central nervous system (Kibleur et al. 2018; Reyt et al. 2010).

\section{Vagus nerve stimulation in inflammatory bowel disease}

Based on the theory of the CAP, we first performed VNS in an experimental model of TNBS (2,4,6-trinitrobenzenesulfonic acid)-induced colitis (Th1-induced inflammation) in rats, resembling CD (Meregnani et al. 2011). VNS was performed in freely moving animals chronically stimulated $3 \mathrm{~h}$ per day for 5 days, starting $1 \mathrm{~h}$ before colitis, with the following stimulation parameters: $1 \mathrm{~mA}$, $5 \mathrm{~Hz}$, pulse width of $500 \mu \mathrm{s} ; 10 \mathrm{~s}$ ON, $90 \mathrm{~s}$ OFF; continuous cycle with an external stimulator. Control rats were implanted according to the same procedure but were not stimulated. VNS reduced body weight loss in rats with colitis and improved inflammatory markers above the colonic lesions as observed by histological score and myeloperoxidase quantification. This anti-inflammatory effect was also demonstrated by the improvement of a multivariate index of colitis (Meregnani et al. 2011).

In a translational bench-to-bedside approach, we performed a pilot study of VNS in patients with active CD (ClinicalTrials.gov Identifier: NCT01569503) where VNS was regarded as an alternative to anti-TNF treatment. This study received a grant from the INSERM-DGOS. Nine patients have been implanted according to the protocol and with the device (neurostimulator and electrode) for epilepsy, using the following parameters: intensity, 0.5-1.5 mA; frequency, $10 \mathrm{~Hz}$; pulse width, $500 \mu \mathrm{s}$; stimulation on-time: $30 \mathrm{~s}$, offtime: $5 \mathrm{~min}$. The first patient was implanted in April 2012 and the last patient in March 2016. Patients were selected according to a clinical activity index (CDAI: Crohn's disease activity index, $220<$ CDAI < 450), biological parameters (C-reactive protein, CRP $>5 \mathrm{mg} / \mathrm{L}$; fecal calprotectin $>$ $100 \mu \mathrm{g} / \mathrm{g}$ ), endoscopic parameters (CDEIS: Crohn's disease endoscopic index of severity; CDEIS $\geq 7$ ) with the aim to induce clinical, biological, and endoscopic remission. It was a 12-month follow-up study. We published, for the first time, the 6-month follow-up of the seven first implanted patients where 5/7 patients had responded to VNS with clinical, biological and endoscopic improvement/healing, with a restored vagal tone (Bonaz et al. 2016). Two patients were withdrawn from the study after 3 months of VNS, due to worsening of their disease. One patient underwent surgery (ileocecal resection) and then was treated with a combination therapy (Infliximab + azathioprine) and the other patient received the same combination therapy. These two patients are presently in remission while the VNS device was left in place with the intensity turned down. Among the other 5 patients, only one patient was treated with an immunosuppressant (azathioprine). These preliminary results showed that VNS was feasible and could be a promising tool in the treatment of active $\mathrm{CD}$. The procedure was safe with the usual side effects described above. No device was removed during the study period and later on. Recently, we published the 12-month follow-up data on 9 patients (Sinniger et al. 2020). After 1 year of VNS, five patients were in clinical remission and six in endoscopic remission. CRP 
and fecal calprotectin decreased in six and five patients, respectively. Seven patients restored their vagal tone and decreased their digestive pain score. The patients' cytokinergic profile evolved toward a more "healthy profile": IL-6, $-23,-12$, TNF and transforming growth factor $\beta 1$ were the most impacted cytokines. Correlations were observed between CRP and TNF, and some gut mucosa metabolites such as taurine, lactate, alanine and beta-hydroxybutyrate. VNS was well tolerated, as reported in the 6-month follow-up study.

These results are in agreement with the preliminary results of another study published in an abstract form (D'Haens et al. 2018), which observed clinical, biomarker, and endoscopic improvement for half of the $16 \mathrm{CD}$ patients who received either VNS monotherapy (biologics refractory patients) or VNS adjunctive therapy for 4 months. We also showed that, in addition to the activation of vagal efferent fibers that regulate the ANS, our data suggest that chronic VNS has a regulatory effect via afferent vagal fibers on anxio-depressive symptomatology associated with $C D$, which could be directly highlighted by the modulation of EEG alpha power known to be associated with depressed states (Kibleur et al. 2018).

\section{VNS in other inflammatory conditions}

RA is also a chronic inflammatory condition where VNS is of interest. Experimental data have shown that knockdown of the $\alpha 7 \mathrm{nAChR}$ in RA fibroblast-like synoviocytes increased the production of mediators of inflammation, and degradation and activation of $\alpha 7 \mathrm{nAChRs}$ in an animal model of RA resulted in reduced arthritis activity (Koopman et al. 2014). Accordingly, stimulation of the CAP by VNS improved an experimental model of arthritis while aggravation of arthritis activity was observed after unilateral cervical vagotomy, as well as in $\alpha 7$ AChR-knockout mice. In 2016, F. A. Koopman et al. (2016) showed - using an implantable device from SetPoint Medical Corporation (Valencia, CA) - that VNS (up to four times daily) significantly inhibited TNF production for up to 84 days in patients with RA. In addition, RA disease severity, measured by standardized clinical composite scores, improved significantly; thus suggesting that it is possible to use neuroimmune modulating device in the therapy of RA with potential implications in other auto-immune and auto-inflammatory diseases. Postoperative ileus (POI) is characterized by a delay of gastric emptying and prolonged intestinal transit after surgery (Stakenborg et al. 2017a). Postoperative ileus can prolong hospitalization and increase healthcare costs. A peripheral pathway, involving the CAP, has shown the release of TNF by resident macrophages in the muscularis propria after abdominal surgery (de Jonge et al. 2003). VNS improves postoperative ileus both in experimental conditions and in humans (Stakenborg et al. 2017b).

\section{Non-invasive vagus nerve stimulation}

The data that we have reported above were based on invasive VNS that requires a surgical implantation of an electrode and a neurostimulator. However, the development of non-invasive VNS techniques is of interest, such as transcutaneous auricular VNS (ta-VNS). This technique is based on the innervation pattern of the lateral surface auricle of the cymba conchae, cavity of the concha, and tragus which are innervated by the auricular (afferent) branch of VN, respectively at $100 \%, 45 \%$, and $45 \%$ (Peuker, Filler 2002). Thus, stimulation of this anatomical part of the auricle will activate vagal afferents targeting the NTS and then activating vagal efferent through the inflammatory reflex described by Tracey's group. Afferent pathway of the $\mathrm{VN}$ and regions activated by taVNS (fMRI studies) are the same (Badran et al. 2018). The Cerbomed Nemos device (Erlangen, Germany) is an external device that provides ta-VNS by using a dedicated intra-auricular electrode (like an earphone) which stimulates the auricular branch of the VN (Stefan et al. 2012). This device received the European clearance (CE mark) in 2010 for epilepsy treatment and is currently available in Germany, Austria, Switzerland, and Italy. Another ta-VNS device is marketed by Schwa Medico (Rouffach, France) with an electrode stimulating the cymba conchae and the cavity of the concha and connected to a neurostimulator (Urostim 2); this device is recommended for IBS (Mion et al. 2020), IBD, RA, migraine, pelvic pain and fibromyalgia treatment. The Electrocore LLC Gammacore device (Basking Ridge, NJ, USA) is a transcutaneous cervical VN stimulator that uses proprietary electrical signals to treat primary headache (Mwamburi et al. 2020). These non-invasive devices are well tolerated with no major side effects, and could be used for the treatment of various inflammatory disorders and others, because the $\mathrm{VN}$ has anti-inflammatory but also anti-nociceptive and anti-depressive properties.

\section{Conclusion}

The anti-inflammatory properties of the $\mathrm{VN}$, both through its afferents (activation of the HPA axis) and efferents (activation of the CAP) can be used to treat various pathological conditions such as chronic inflammatory diseases. In particular, 
as a key element of the ANS in the brain-gut interactions in IBD (Bonaz et al. 2013), the VN is a therapeutic target in IBD. In addition, the $\mathrm{VN}$ is also at the interface of the microbiota-gut-brain axis (Bonaz et al. 2018) and based on the implication of dysbiosis in various pathological conditions, one can assume that VNS is also able to target this dysbiosis. VNS is able to restore vagal tone. Indeed, we have reported an abnormal ANS in IBD patients (Pellissier et al. 2010) negatively correlated with TNF $\alpha$ levels (Pellissier et al. 2014). VNS, by restoring the ANS balance in such patients through the activation of the VN, is a novel therapeutic treatment. Finally, VNS is devoid of the adverse events of biological therapies such as anti-TNF drugs (infections, dermatological complications, lymphoma, immunisation) which are still a gold stan- dard treatment in IBD but are feared by patients, thus explaining their non-adherence to treatment and their search for a non-drug therapy. Finally, the economic impact of VNS is of interest since, for example, the median cost of 1-year anti-TNF therapy raises up to $\$ 40000$ for CD patients (Targownik et al. 2019). The use of neuromodulation by bioelectronics devices as a treatment is an emerging field in the domain of bioelectronic medicine. It could be an alternative non-drug therapy to conventional treatment or could be combined with such treatments, but further investigation in large longitudinal cohorts of patients is required. Finally, the optimal neurostimulation parameters to better treat common conditions and diseases that involve immune regulation to achieve significant cytokine changes need to be determined (Bonaz 2020).

\section{References}

Abe, C., Inoue, T., Inglis, M. A. et al. (2017) C1 neurons mediate a stress-induced anti-inflammatory reflex in mice. Nature Neuroscience, vol. 20, no. 5, pp. 700-707. DOI: 10.1038/nn.4526 (In English)

Altschuler, S. M., Escardo, J., Lynn, R. B., Miselis, R. R. (1993) The central organization of the vagus nerve innervating the colon of the rat. Gastroenterology, vol. 104, no. 2, pp. 502-509. DOI: 10.1016/0016-5085(93)90419-d (In English)

Badran, B. W., Dowdle, L. T., Mithoefer, O. J. et al. (2018) Neurophysiologic effects of transcutaneous auricular vagus nerve stimulation (taVNS) via electrical stimulation of the tragus: A concurrent taVNS/fMRI study and review. Brain Stimulation, vol. 11, no. 3, pp. 492-500. DOI: 10.1016/j.brs.2017.12.009 (In English)

Benarroch, E. E. (1993) The central autonomic network: Functional organization, dysfunction, and perspective. Mayo Clinic Proceedings, vol. 68, no. 10, pp. 988-1001. DOI: 10.1016/s0025-6196(12)62272-1 (In English)

Bernik, T. R., Friedman, S. G., Ochani, M. et al. (2002) Cholinergic anti-inflammatory pathway inhibition of tumor necrosis factor during ischemia reperfusion. Journal of Vascular Surgery, vol. 36, no. 6, pp. 1231-1236. DOI: 10.1067/mva.2002.129643 (In English)

Berthoud, H. R., Carlson, N. R., Powley, T. L. (1991) Topography of efferent vagal innervation of the rat gastrointestinal tract. American Journal of Physiology-Regulatory, Integrative and Comparative Physiology, vol. 260, no. 1, pp. R200-R207. DOI: 10.1152/ajpregu.1991.260.1.R200 (In English)

Bonaz, B. (2020) Parameters matter: Modulating cytokines using nerve stimulation. Bioelectronic Medicine, vol. 6, article 12. DOI: 10.1186/s42234-020-00049-1 (In English)

Bonaz, B., Bazin, T., Pellissier, S. (2018) The vagus nerve at the interface of the microbiota-gut-brain axis. Frontiers in Neuroscience, vol. 12, article 49. DOI: 10.3389/fnins.2018.00049 (In English)

Bonaz, B. L., Bernstein, C. N. (2013) Brain-gut interactions in inflammatory bowel disease. Gastroenterology, vol. 144, no. 1, pp. 36-49. DOI: 10.1053/j.gastro.2012.10.003 (In English)

Bonaz, B., Lane, R. D., Oshinsky, M. L. (2020) Diseases, disorders, and comorbidities of interoception. Trends in Neurosciences. (In press). (In English)

Bonaz, B., Sinniger, V., Hoffmann, D. et al. (2013) Vagus nerve stimulation: From epilepsy to the cholinergic antiinflammatory pathway. Journal of Neurogastroenterology and Motility, vol. 25, no. 3, pp. 208-221. DOI: 10.1111/nmo.12076 (In English)

Bonaz, B., Sinniger, V., Hoffmann, D. et al. (2016) Chronic vagus nerve stimulation in Crohn's disease: A 6-month follow-up pilot study. Journal of Neurogastroenterology and Motility, vol. 28, no. 6, pp. 948-953. DOI: $10.1111 /$ nmo.12792 (In English)

Bonaz, B., Sinniger, V., Pellissier, S. (2017) The vagus nerve in the neuro-immune axis: Implications in the pathology of the gastrointestinal tract. Frontiers in Immunology, vol. 8, article 1452. DOI: 10.3389/fimmu.2017.01452 (In English)

Bonaz, B., Sinniger, V., Pellissier, S. (2019) Vagus nerve stimulation at the interface of brain-gut interactions. Cold Spring Harbor Perspectives in Medicine, vol. 9, no. 8, article a034199. DOI: 10.1101/cshperspect.a034199 (In English)

Borovikova, L. V., Ivanova, S., Zhang, M. et al. (2000) Vagus nerve stimulation attenuates the systemic inflammatory response to endotoxin. Nature, vol. 405, no. 6785, pp. 458-462. DOI: 10.1038/35013070 (In English) 
Buller, K. M. (2001) Role of circumventricular organs in pro-inflammatory cytokine-induced activation of the hypothalamic-pituitary-adrenal axis. Clinical and Experimental Pharmacology and Physiology, vol. 28, no. 7, pp. 581-589. DOI: 10.1046/j.1440-1681.2001.03490.x (In English)

Cailotto, C., Gomez-Pinilla, P. J., Costes, L. M. et al. (2014) Neuro-anatomical evidence indicating indirect modulation of macrophages by vagal efferents in the intestine but not in the spleen. PLoS One, vol. 9, no. 1, article e87785. DOI: 10.1371/journal.pone.0087785 (In English)

Cechetto, D. F. (1987) Central representation of visceral function. Federation Proceedings, vol. 46, no. 1, pp. 17-23. PMID: 3542576. (In English)

Chan, W., Chen, A., Tiao, D. et al. (2017) Medication adherence in inflammatory bowel disease. Intestinal Research, vol. 15, no. 4, pp. 434-445. DOI: 10.5217/ir.2017.15.4.434 (In English)

Click, B., Regueiro, M. (2019) Managing risks with biologics. Current Gastroenterology Reports, vol. 21, no. 2, article 1. DOI: 10.1007/s11894-019-0669-6 (In English)

De Jonge, W. J., van den Wijngaard, R. M., The, F. O. et al. (2003) Postoperative ileus is maintained by intestinal immune infiltrates that activate inhibitory neural pathways in mice. Gastroenterology, vol. 125, no. 4, pp. 11371147. DOI: 10.1016/s0016-5085(03)01197-1 (In English)

De Jonge, W. J., van der Zanden, E. P., The, F. O. et al. (2005) Stimulation of the vagus nerve attenuates macrophage activation by activating the Jak2-STAT3 signaling pathway. Nature Immunology, vol. 6, no. 8, pp. 844-851. DOI: 10.1038/ni1229 (In English)

Delmas, J., Laux, G. (1933) Anatomie médico-chirurgicale du système nerveux végétatif: sympathique \& parasympathique [Medical-surgical anatomy of the vegetative nervous system: sympathetic and parasympathetic]. Paris: Masson, 266 p. (In French)

D'Haens, G. R., Cabrijan, Z., Eberhardson, M. (2018) The effects of vagus nerve stimulation in biologic-refractory Crohn's disease: A prospective clinical trial. Journal of Crohn's and Colitis, vol. 12, no. 1, pp. S397-S398. DOI: 10.1093/ecco-jcc/jjx180.701 (In English)

Englot, D. J., Chang, E. F., Auguste, K. I. (2011) Vagus nerve stimulation for epilepsy: A meta-analysis of efficacy and predictors of response. Journal of Neurosurgery, vol. 115, no. 6, pp. 1248-1255. DOI: 10.3171/2011.7.JNS11977 (In English)

Fournier, A., Mondillon, L., Luminet, O. et al. (2020) Interoceptive abilities in inflammatory bowel diseases and irritable bowel syndrome. Frontiers in Psychiatry, vol. 11, article 229. DOI: 10.3389/fpsyt.2020.00229 (In English)

Ghia, J. E., Blennerhassett, P., Kumar-Ondiveeran, H. et al. (2006) The vagus nerve: A tonic inhibitory influence associated with inflammatory bowel disease in a murine model. Gastroenterology, vol. 131, no. 4, pp. 1122-1130. DOI: 10.1053/j.gastro.2006.08.016 (In English)

Kibleur, A., Pellissier, S., Sinniger, V. et al. (2018) Electroencephalographic correlates of low-frequency vagus nerve stimulation therapy for Crohn's disease. Clinical Neurophysiology, vol. 129, no. 5, pp. 1041-1046. DOI: 10.1016/j. clinph.2018.02.127 (In English)

Koopman, F. A., Chavan, S. S., Miljko, S. et al. (2016) Vagus nerve stimulation inhibits cytokine production and attenuates disease severity in rheumatoid arthritis. Proceedings of the National Academy of Sciences of USA, vol. 113, no. 29, pp. 8284-8289. DOI: 10.1073/pnas.1605635113 (In English)

Koopman, F. A., Schuurman, P. R., Vervoordeldonk, M. J., Tak, P. P. (2014) Vagus nerve stimulation: A new bioelectronics approach to treat rheumatoid arthritis? Best Practice \& Research Clinical Rheumatology, vol. 28, no. 4, pp. 625-635. DOI: 10.1016/j.berh.2014.10.015 (In English)

Krahl, S. E., Senanayake, S. S., Handforth, A. (2001) Destruction of peripheral C-fibers does not alter subsequent vagus nerve stimulation-induced seizure suppression in rats. Epilepsia, vol. 42, no. 5, pp. 586-589. DOI: 10.1046/j.1528-1157.2001.09700.x (In English)

Martelli, D., McKinley, M. J., McAllen, R. M. (2014) The cholinergic anti-inflammatory pathway: A critical review. Autonomic Neuroscience: Basic \& Clinical, vol. 182, pp. 65-69. DOI: 10.1016/j.autneu.2013.12.007 (In English)

Meregnani, J., Clarencon, D., Vivier, M. et al. (2011) Anti-inflammatory effect of vagus nerve stimulation in a rat model of inflammatory bowel disease. Autonomic Neuroscience: Basic \& Clinical, vol. 160, no. 1-2, pp. 82-89. DOI: 10.1016/j.autneu.2010.10.007 (In English)

Mion, F., Pellissier, S., Garros, A. et al. (2020) Transcutaneous auricular vagus nerve stimulation for the treatment of irritable bowel syndrome: A pilot, open-label study. Neuromodulation. (In press). (In English)

Morris, G. L., Mueller, W. M., The Vagus Nerve Stimulation Study Group E01-E05. (1999) Long-term treatment with vagus nerve stimulation in patients with refractory epilepsy. Neurology, vol. 53, no. 8, pp. 1731-1735. DOI: 10.1212/wnl.53.8.1731 (In English)

Mwamburi, M., Liebler, E. J., Staats, P. S. (2020) Patient experience with non-invasive vagus nerve stimulator: GammaCore patient registry. American Journal of Managed Care, vol. 26, no. 1, pp. S15-S19. DOI: 10.37765/ajmc.2020.42545 (In English)

Netter, F. H., Colacino, S. (1989) Atlas of human anatomy. Summit: Ciba-Geigy Corporation, 550 p. (In English)

Olofsson, P. S., Katz, D. A., Rosas-Ballina, M. et al. (2012) $\alpha$-7-Nicotinic acetylcholine receptor ( $\alpha 7 \mathrm{nAChR}$ ) expression in bone marrow-derived non-T cells is required for the inflammatory reflex. Molecular Medicine, vol. 18, pp. 539-543. DOI: 10.2119/molmed.2011.00405 (In English) 
Pellissier, S., Dantzer, C., Canini, F. et al. (2010) Psychological adjustment and autonomic disturbances in inflammatory bowel diseases and irritable bowel syndrome. Psychoneuroendocrinology, vol. 35, no. 5, pp. 653-662. DOI: 10.1016/j.psyneuen.2009.10.004 (In English)

Pellissier, S., Dantzer, C., Mondillon, L. et al. (2014) Relationship between vagal tone, cortisol, TNF-alpha, epinephrine and negative affects in Crohn's disease and irritable bowel syndrome. PLoS One, vol. 9, no. 9, article e105328. DOI: 10.1371/journal.pone.0105328 (In English)

Peuker, E. T., Filler, T. J. (2002) The nerve supply of the human auricle. Clinical Anatomy, vol. 15, no. 1, pp. 35-37. DOI: 10.1002/ca.1089 (In English)

Reyt, S., Picq, C., Sinniger, V. et al. (2010) Dynamic causal modelling and physiological confounds: A functional MRI study of vagus nerve stimulation. Neuroimage, vol. 52, no. 4, pp. 1456-1464. DOI: 10.1016/j.neuroimage.2010.05.021 (In English)

Ricardo, J. A., Koh, E. T. (1978) Anatomical evidence of direct projections from the nucleus of the solitary tract to the hypothalamus, amygdala, and other forebrain structures in the rat. Brain Research, vol. 153, no. 1, pp. 1-26. DOI: 10.1016/0006-8993(78)91125-3 (In English)

Rosas-Ballina, M., Ochani, M., Parrish, W. R. et al. (2008) Splenic nerve is required for cholinergic antiinflammatory pathway control of TNF in endotoxemia. Proceedings of the National Academy of Sciences of USA, vol. 105, no. 31, pp. 11008-11013. DOI: 10.1073/pnas.0803237105 (In English)

Sinniger, V., Pellissier, S., Fauvelle, F. et al. (2020) A 12-month pilot study outcomes of vagus nerve stimulation in Crohn's disease. Neurogastroenterology \& Motility, vol. 32, no. 10, article e13911. DOI: 10.1111/nmo.13911 (In English)

Stakenborg, N., Gomez-Pinilla, P. J., Boeckxstaens, G. E. (2017a) Postoperative ileus: Pathophysiology, current therapeutic approaches. Gastrointestinal Pharmacology. Handbook of Experimental Pharmacology, vol. 239, pp. 39-57. DOI: 10.1007/164_2016_108 (In English)

Stakenborg, N., Wolthuis, A. M., Gomez-Pinilla, P. J. et al. (2017b) Abdominal vagus nerve stimulation as a new therapeutic approach to prevent postoperative ileus. Neurogastroenterology \& Motility, vol. 29, no. 9, article e13075. DOI: 10.1111/nmo.13075 (In English)

Stefan, H., Kreiselmeyer, G., Kerling, F. et al. (2012) Transcutaneous vagus nerve stimulation (t-VNS) in pharmacoresistant epilepsies: A proof of concept trial. Epilepsia, vol. 53, no. 7, pp. e115-e118. DOI: 10.1111/j.1528-1167.2012.03492.x (In English)

Strack, A. M., Sawyer, W. B., Platt, K. B., Loewy, A. D. (1989) CNS cell groups regulating the sympathetic outflow to adrenal gland as revealed by transneuronal cell body labelling with pseudorabies virus. Brain Research, vol. 491, no. 2, pp. 274-296. DOI: 10.1016/0006-8993(89)90063-2 (In English)

Targownik, L. E., Benchimol, E. I., Witt, J. et al. (2019) The effect of initiation of anti-TNF therapy on the subsequent direct health care costs of inflammatory Bowel disease. Inflammatory Bowel Diseases, vol. 25, no. 10, pp. 1718-1728. DOI: 10.1093/ibd/izz063 (In English)

Torres, J., Ellul, P., Langhorst, J. et al. (2019) European Crohn's and colitis organisation topical review on complementary medicine and psychotherapy in inflammatory Bowel disease. Journal of Crohn's and Colitis, vol. 13, no. 6, pp. 673-685e. DOI: 10.1093/ecco-jcc/jjz051 (In English)

Wang, H., Liao, H., Ochani, M. et al. (2004) Cholinergic agonists inhibit HMGB1 release and improve survival in experimental sepsis. Nature Medicine, vol. 10, no. 11, pp. 1216-1221. DOI: 10.1038/nm1124 (In English)

Wang, H., Yu, M., Ochani, M. et al. (2003) Nicotinic acetylcholine receptor $\alpha 7$ subunit is an essential regulator of inflammation. Nature, vol. 421, no. 6921, pp. 384-388. DOI: 10.1038/nature01339 (In English)

Werner, M. F. P., Fraga, D., Melo, M. C. C. et al. (2003) Importance of the vagus nerve for fever and neutrophil migration induced by intraperitoneal LPS injection. Inflammation Research, vol. 52, no. 7, pp. 291-296. DOI: 10.1007/s00011-003-1174-8 (In English)

Zagon, A. (2001) Does the vagus nerve mediate the sixth sense? Trends in Neurosciences, vol. 24, no. 11, pp. 671-673. DOI: 10.1016/s0166-2236(00)01929-9 (In English) 